\title{
Addictive agents and intracranial stimulation (ICS): Daily morphine, self-stimulation, and parameters of ICS
}

\author{
LORETTA TERANDO, NOOR MIRZA, JAN ZIPNICK, MARY OVERMIER, \\ NELLO ROSSI, and LARRY D. REID \\ Bradley University, Peoria, Illinois 61606
}

\begin{abstract}
Rats fixed with chronically indwelling bipolar electrodes pressed daily for direct electrical intracranial stimulation (ICS) of the hypothalamus. Rats pressed for a variety of intensities of ICS. As daily sessions with ICS continued, rats were given doses of morphine sulfate. The data confirm that morphine can accelerate pressing for ICS regardless of the intensities of ICS. One set of procedures indicated that programming ICS of about $75 \mu \mathrm{A}(60-\mathrm{Hz}$ sine waves of $.25 \mathrm{sec}$ ) was sufficient to disrupt pressing previously sustained by intensities of $25 \mu \mathrm{A}$ or lower. There were considerable individual differences in rats' reactivity to morphine; some showed marked acceleration of pressing while others showed little or no acceleration. These differences were not a direct function of intensity of ICS.
\end{abstract}

The medial forebrain bundle (MFB) system of the upper brainstem is apparently critical to reinforcement (Olds, 1969). In subjects fixed with chronically indwelling electrodes, a measure of the functional characteristics of the MFB is rate of responding for MFB intracranial stimulation (ICS). Because addictive agents might modify reinforcement processes (Collier, 1969), it is of interest to know how a prototypic addictive agent such as morphine might affect the leverpressing for MFB stimulation.

The effects of 10 - to $20-\mathrm{mg} / \mathrm{kg}$ daily doses of morphine on pressing for a fixed intensity of hypothalamic ICS can now be described roughly (Adams, Lorens, \& Mitchell, 1972; Bush, Bush, Miller, \& Reid, 1976; Lorens \& Mitchell, 1973; Olds \& Travis, 1960; Pert \& Hulsebus, 1974). Rate of pressing is determined by a complex interaction of Dose by Time After Dosing by Days of Daily Dosing. Pressing is depressed shortly after injections (about $1 \mathrm{~h}$ ), but is facilitated subsequently (4 to $6 \mathrm{~h}$ ). With continuance of daily doses, the extent of suppression of pressing just following injections is reduced and the facilitation is seen sooner and sooner after injections. Morphine's characteristic action on pressing for ICS supports the conclusion that morphine might have an important site of action within the MFB system.

The next problems that were addressed were whether or not morphine's characteristic effects were

This research was supported by Grant DA01049, from the National Institute on Drug Abuse, DHEW. A more complete report of research mentioned in the introduction appears in Terando (1974). We thank Cathy Kaczynski for help with this research. Correspondence should be addressed to L. Reid, who is now at Department of Psychology, Rensselaer Polytechnic Institute, Troy, New York 12181. seen across a range of intensities of ICS. A series of three tests of morphine's effects across a range of intensities produced confusing results with respect to responding after termination of all injections. The first test indicated that morphine did accelerate pressing across a range of intensities of ICS under certain regimens of testing. The test also indicated, however, that press rates were considerably depressed subsequent to daily morphine injections. The next two experiments involved further testing with morphine and a range of intensities of ICS. Neither test indicated that rats' pressing was depressed following termination of morphine injections. The procedures of the three tests were similar except for the upper limit of the range of intensities of ICS. The first test involved ranges of ICS up to twice the microamperes of the second and third tests. The higher intensities of ICS of the first test were, perhaps, sufficiently high to have caused the decreased pressing seen subsequent to morphine. The following experiment was done to compare the effects of using higher and lower ranges of intensities, to see if the critical difference among the previous experiments could have been due to the upper limits of the range of intensities of ICS.

\section{EXPERIMENT 1}

\footnotetext{
Method

Subjects. Sixteen experimentally naive, adult, male, SpragueDawley rats completed the procedures. All were fixed with chronically indwelling electrodes. The electrodes (Plastic Products) were bipolar stainless steel wires insulated except at the cross section of the tips. The stimulating tips were separated from one another only by the width of the insulation. Following recovery from surgery and preliminary training, all pressed a lever for ICS at intensities of 40 to $50 \mu \mathrm{A}$. Rats were housed in individual cages in a
} 
room without windows and with constant lighting. Food and water was always available to the rats.

Apparatus. The test chamber (a $24 \times 30 \times 35 \mathrm{~cm}$ box) contained a lever, the depression of which yielded ICS. ICS leads were light, flexible wires attached to a slip ring assembly allowing unhampered movement by the rat. Leverpresses during 2-min periods were recorded automatically. The ICS was $60-\mathrm{Hz}$ sine waves of $0.3 \mathrm{sec}$. Intensity of ICS was measured peak to peak of the wave.

Procedure. Following surgery, recovery from surgery, and tests to confirm that rats pressed for ICS, each rat was tested daily in the late afternoon with four intensities of ICS, 10, 25, 40, and $50 \mu \mathrm{A}$. Tests always started with the lowest intensity and ended with the highest intensity. Before each 2-min test with a particular intensity of ICS, the rat was allowed to take at least 10 self-delivered ICSs prior to measuring rate of pressing at that intensity, or, if a rat failed to respond, it was given 10 ICSs while being shaped toward pressing. Throughout training and testing, each leverpress yielded a single 0.3-sec train of ICS.

Subsequent to the rats' pressing becoming somewhat stable across daily tests (at least 10 days of tests), there were 25 consecutive days of further testing. The 25 days of testing were divided into five blocks of 5 days each. For the first block of tests, all 16 rats pressed daily for each of the four intensities of $\operatorname{ICS}(10,25$, 40 , and $50 \mu \mathrm{A})$. As would be expected, rats pressed least for $10 \mu \mathrm{A}$ and most for 40 and $50 \mu \mathrm{A}$. For the next 5 days of tests (Block 2), one-half of the rats $(n=8)$ continued as during Block 1 testing. The other half of the rats were introduced to a new set of ICS intensities $(10,25,75$, and $100 \mu \mathrm{A})$.

During the second block of tests, there were two groups of rats, one getting lower intensities of ICS and one getting higher intensities of ICS. During the third and fourth blocks of testing, onehalf of each group were given morphine sulfate $(10 \mathrm{mg} / \mathrm{kg})$ and one-half a placebo (the carrier of morphine, isotonic water). All injections were $4 \mathrm{~h}$ prior to daily tests with ICS. The intensities remained the same as during the second block of tests. Consequently, there were four groups of rats, four to a group. The procedure of daily injections and tests with ICS continued for two blocks of 5 days each (Blocks 3 and 4). Then all injections were terminated and a final 5-day block of tests with ICS (Block 5) were completed while intensities remained as during Blocks 2, 3, and 4.

Subsequent to the procedure measuring pressing, nearly all subjects' brains of this experiment and the other tests reported here were examined to determine more exactly the site of ICS. Frozen 50- to $70-\mu$ sections of the brain were treated as photographic negatives, and an enlarged image of sections of the electrode tract were photographed to aid in determining site of ICS.

\section{Results and Discussion}

Results of the first two blocks of tests are depicted in Figure 1. Using the mean number of presses during a block at each intensity of ICS as the "raw data," an ANOVA was calculated for the data used to determine the points of Figure 1. The ANOVA followed a 2 by 2 by 4 design, with repeated measures on the last two factors. One factor was the two groups of rats, one group of only lower intensities and one group of higher intensities during Block 2 . The other two factors were associated with the two blocks and the four intensities of ICS.

The main effect associated with intensity was, of course, a reliable source of variance $[F(3,42)=$ $55.4, \mathrm{p}<.01]$; no other main effect was a reliable source of variance ( $\mathrm{ps}>.10)$. The Groups by Intensities interaction was reliable $[\mathrm{F}(3,42)=4.8, \mathrm{p}<.01]$,

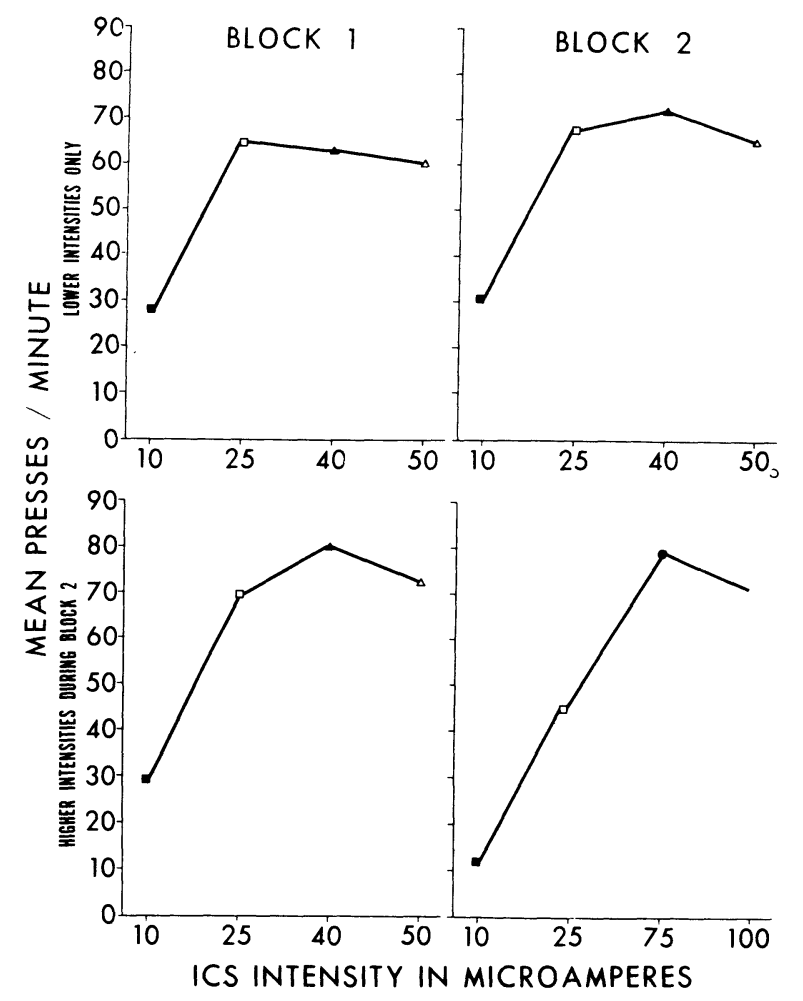

Figure 1. Block means for each intensity of ICS. Block 1 pressing is for only lower intensities of ICS. Block 2 pressing is for both pressing for lower (upper panel) and higher intensities of ICS (lower panel).

as were the Groups by Block interaction $[F(1,14)=$ $20.5, \mathrm{p}<.01]$ and the Blocks by Intensity interaction $[F(3,42)=5.9, p<.01]$. The triple interaction yielded an $F(3,42)=2.6, p<.10$. Results of the ANOVA allow the specific tests of the hypothesis that introduction of the higher intensities of ICS led to less pressing.

Student $t$ tests for correlated measures were used to determine reliability of differences in pressing across Block 1 to Block 2 within a group, and they yielded the following values: $t(7)$, rats of lower intensity, $=-2.7, p<.05 ; t(7)$, rats of higher intensity, $=3.7, \mathrm{p}<.01$. As typically seen (e.g., Bush et al., 1976), rats that merely continued with daily sessions with ICS, as did the rats of only lower intensities, increased their pressing rates. On the other hand, the rats that received higher intensities, decreased their rate of pressing after introduction of the higher intensities.

As can be seen in Figure 1, the greatest reduction in pressing rates for rats that were introduced to higher intensities occurred with the two lower intensities. To summarize those shifts, $t$ tests were done comparing the data of Blocks 1 and 2. For rats of high intensity and with pressing at $10 \mu \mathrm{A}, \mathrm{t}(7)=2.8$, $\mathrm{p}<.05$. For rats of high intensity and with pressing 
at $25 \mu \mathrm{A}, \mathrm{t}(7)=4.2, \mathrm{p}<.01$. Comparable t tests for rats of low intensity yielded $t$ values opposite in sign, with $p$ values $>.10$. In other words, rats introduced to high intensities reduced their pressing at the two lower intensities by more than $40 \%$ while the rats not introduced to higher intensities increased their pressing at these intensities by $\mathbf{8 \%}$. The conclusion is that introduction of higher intensities reduced pressing, particularly at the lower range of intensities that will sustain pressing prior to introduction of higher intensities.

The data of blocks of injections for rats of low intensity and of high intensity were analyzed separately. Each ANOVA followed a 2 by 2 by 4 factorial design with repeated measures on the last two factors. One factor was associated with the groups of rats (morphine-saline, $n=4 /$ group), one factor with the two 5-day blocks, and the other with the four intensities of ICS. The scores submitted to the ANOVA were difference scores, i.e., a rat's scores were the mean of a block for a particular intensity minus the comparable mean of Block 2. Consequently, scores greater than 0.0 indicate that pressing increased from Block 2 pressing, while scores less than 0.0 indicate decreased pressing.

The results of tests, in difference scores, of Blocks 3,4 , and 5 are given in Figure 2. The ANOVA for the data of the low-intensity procedures (two upper left panels, Figure 2) yielded reliable values for the main effects of blocks $[F(1,6)=6.4, p<.05]$ and intensities $[\mathrm{F}(3,18)=5.9, \mathrm{p}<.01]$ and the interaction of Blocks by Intensities $[F(3,18)=3.4, p<.05]$. Factors associated with morphine-saline were not reliable sources of variance.

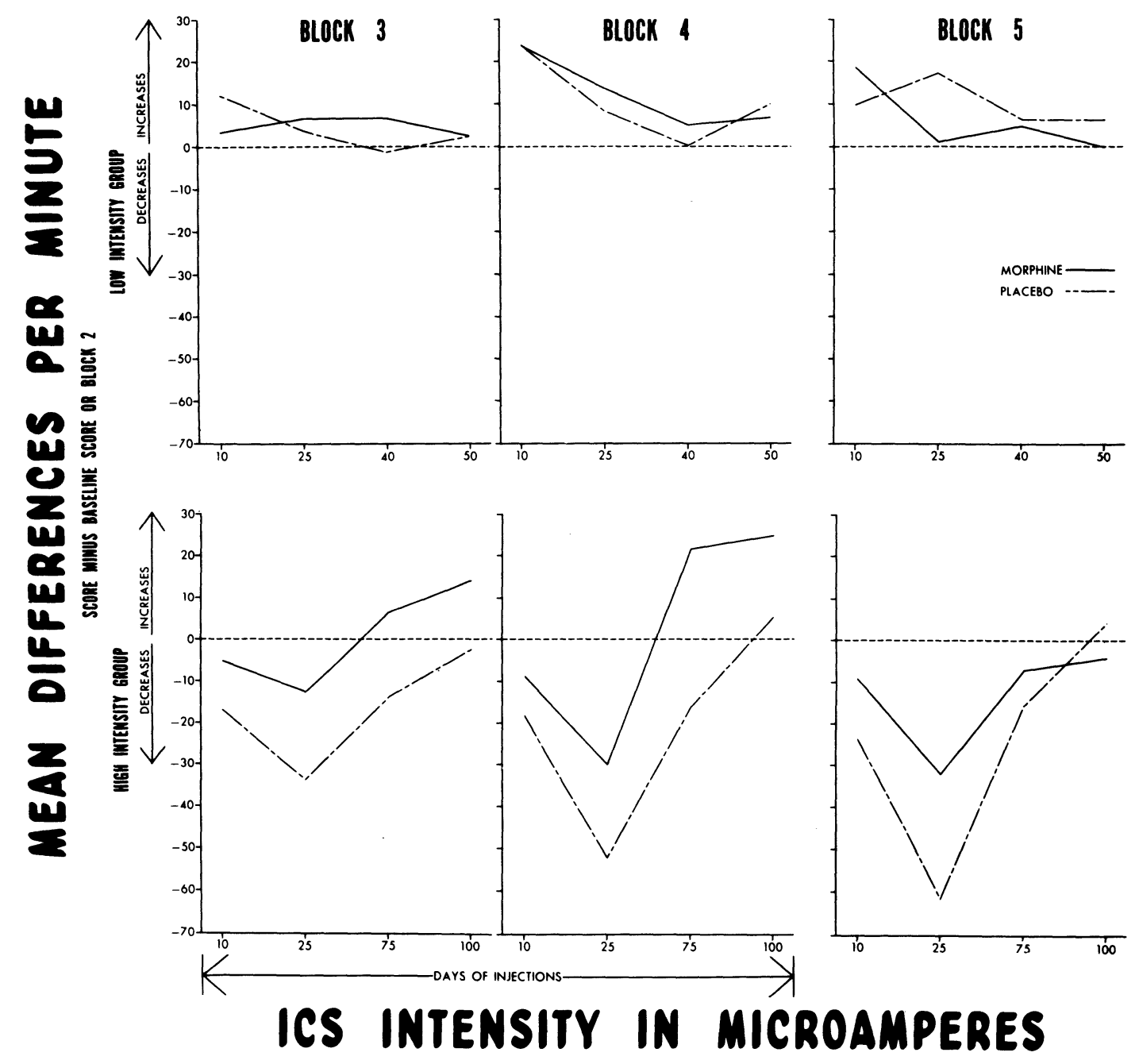

Figure 2. Mean difference scores for each block of tests at each intensity of ICS. A difference score was mean pressing score minus comparable mean score during Block 2. During Blocks 3 and 4 , one-half of the rats got $10 \mathrm{mg} / \mathrm{kg}$ of morphine sulfate, $4 \mathrm{~h}$ prior to testing. 
Although the ANOVA indicates no reliable differences associated with morphine-saline effects, certain tests are dictated by previous results (Bush et al., 1976). Student $t$ tests for correlated measures were calculated for scores of both saline and morphine rats between baseline (Block 2) and the second block of morphine (Block 4) at 25 and $40 \mu \mathrm{A}$. These scores and tests provide a replication of Bush et al. Only the $t$ for the difference between baseline and Block 4 scores of rats of morphine at $25 \mu \mathrm{A}$ was great enough to conclude a reliable increase in pressing, $t(3)=4.3$, $\mathrm{p}<.05$. These results, plus general trends in the data, provide weak confirmation for the conclusion of Bush et al. that morphine facilitates pressing at moderate intensities of ICS, and leaves open the question of whether or not morphine facilitates pressing all along the range of intensities below the "lesion-producing"' higher intensities.

Results of tests with the higher intensities are depicted in the two left panels of the lower row of Figure 2. The ANOVA for scores of rats of the higher intensities during days of injections yields an $F(1,6)=20.6, p<.01$, for the factor associated with morphine-saline; an $F(3,18)=15.6, p<.01$, for the factor of intensity; and an $F(3,18)=4.5$, $\mathrm{p}<.05$, for the interaction of Blocks by Intensity. Other factors were not reliable sources of variance.

Analysis of the postinjection block of scores for rats of lower intensities suggests no reliable difference between groups (morphine-saline) or across intensities (all Fs $<1.8$ ). Since one rat of the highintensity/morphine group lost its electrode midway during Block 5 testing, results of the figure and the analyses are for only three rats for that group. A repeated measures ANOVA of postinjection scores for rats of higher intensities suggests no difference between groups $(F<1)$, but reliable effects across intensities $[\mathrm{F}(3,15)=10.1, \mathrm{p}<.01]$.

The data of Figure 2 and the accompanying analyses are difference scores. Because they are difference scores, it was not expected that there would be as strong intensity effects as you would expect with direct data of press rates. Yet, analyses of the data yielded reliable main effects of intensity. With the low-intensity group, pressing at the lower intensities tended to increase across blocks of testing, thereby yielding fairly large positive difference scores. With the high-intensity group, pressing at lower intensities tended to decrease across blocks of testing, theraby yielding fairly large negative difference scores. With both the low- and high-intensity groups, pressing at the higher intensities tended to be about the same across blocks of testing, thereby yielding small difference scores.

Inspection of sections of the brains of rats of this and previous procedures indicated that all electrode tips were within the area of the lateral hypothalamus.
Electrode tips were somewhat scattered about the MFB and always lateral to the fornix columns, usually in the same anterior-posterior plane as the ventromedial $\mathrm{n}$. Photographs of brain sections of rats exposed to only lower intensities failed to show any tissue damage at the electrode tip. Sections of brain exposed to higher intensities did seem to show some necrotic tissue at the electrode tip. The difference between the two kinds of sections was, however, not as marked as might be expected. Perhaps more microscopic examination might reveal differences not apparent with the techniques used here which involved only 10 times magnification.

The first part of these procedures (Figure 1) demonstrated that introduction of intensities of from 75 to $100 \mu \mathrm{A}$ reduced the capacity of lower intensities to maintain high rates of pressing. Presumably, this effect is due to lesions produced at the electrode tip by the higher intensities of ICS (Valenstein \& Beer, 1961). Despite evidence of lesions (reduced responding at lower intensities), the higher intensities maintained high rates of pressing. Ellman, Ackerman, Bodnar, Jackler, and Steiner (1975), using an ICS very similar to that used here $(60-\mathrm{Hz}$ sine waves of $0.25 \mathrm{sec}$ ), determined press rates by intensity functions using intensities of 100 and $200 \mu \mathrm{A}$ (in one procedure as much as $500 \mu \mathrm{A}$ ). From data of procedures reported here, and also demonstrated in an experiment of Magnuson, Tadeusik, and Reid (1976), it is clear that these intensities are sufficient to disrupt pressing at the lower intensities. Furthermore, our data indicate that shifts in pressing continue to occur across days of stimulation with the higher intensities of ICS. We conclude, therefore, that results of Ellman et al. (as well as data of our initial studies) were compromised by the lesions most likely to have occurred with introduction of the higher intensities of ICS.

The second part of our procedures involving data of days of injections of morphine and placebo demonstrate that quite different results are obtainable when only lower intensities are used compared to when higher intensities have been introduced. If the only available data concerning morphine's effects on pressing for ICS were those of this experiment using only lower intensities, the proper conclusion would be that morphine had no reliable facilitatory effects. On the other hand, if the only data available were those of higher intensities, it could be concluded that morphine had powerful facilitatory effects compared to that of a placebo.

\section{EXPERIMENT 2}

In Experiment 1, marked facilitation of pressing for ICS under the influence of morphine occurred only with rats tested with "lesion-producing" inten- 
sities of ICS (ICS greater than $75 \mu \mathrm{A}$ of $60-\mathrm{Hz}$ sine waves of $.25 \mathrm{sec}$ ), and the facilitation did not clearly occur in rats tested with ICS of $50 \mu \mathrm{A}$ or less. This result left open the question as to whether morphine accelerated pressing regardless of intensity of ICS. Since the results of Experiment 1 raised a question that could not be resolved in the extant literature, and since the findings of Experiment 1 are limited by both small group size $(n=4)$ and slight facilitation of pressing with the group of placebos, this experiment addressed the question of whether morphine accelerates pressing at various intensities below "lesion-producing" intensities.

\section{Method}

Subjects and Apparatus. The subjects were 28 experimentally naive, adult, male, Sprague-Dawley rats. Each was fixed, in standard ways, with the same type of electrode as rats of Experiment 1. During initial testing, all rats pressed a lever more than 40 times/ min for ICS of $50 \mu \mathrm{A}$ or less $(60-\mathrm{Hz}$ sine waves of $0.25 \mathrm{sec})$. The subjects were housed individually with food and water always available. The rats were tested daily in the same apparatus as used in Experiment 1.

Procedure. Subsequent to surgery, recovery from surgery (spanning at least 5 days), and initial testing with ICS, each rat was tested daily with ICS. During the first days of testing, ICS intensities were varied and four intensities of ICS were selected for each subject, zero, low, medium, and high ICS. The low-intensity ICS was near the rat's lower threshold intensity for maintaining pressing. The high intensity was an ICS that sustained rapid steady pressing. Medium ICS was about in the middle between the other two. The limits of the ranges of intensities were 5 to $25 \mu \mathrm{A}$ for low, 10 to $30 \mu \mathrm{A}$ for medium, and 15 to $40 \mu \mathrm{A}$ for high.

After selecting the intensities, the rats were tested the same way daily under a similar regimen as were the rats of Experiment 1. During each daily session, a rat was allowed to press at each intensity for $2 \mathrm{~min}$, starting with zero intensity and ending with high intensity, while number of presses were recorded. Each 2-min measurement was preceded by a period in which the subjects were either shaped to press by 10 ICSs of the appropriate intensity or they were allowed to press by themselves to become acquainted with the intensity of ICS.

After the rats had had at least a week of daily sessions to stabilize pressing for selected ICS intensities, 2 days of preinjection baseline data were collected. Then daily subcutaneous injections of morphine sulfate, $10 \mathrm{mg} / \mathrm{kg}$, were given to 19 of the subjects for 3 consecutive days; the other 9 subjects received placebos (physiological saline injections of the same volume as the morphine injections). The injections were given $4 \mathrm{~h}$ prior to measurements with ICS.

\section{Results and Discussion}

Figure 3 depicts the mean presses $/ 2$ min of the morphine and placebo groups at each intensity of ICS during baseline immediately prior to injections. As can be seen, means for the two groups hardly differed prior to treatment. Also, pressing for ICS was rapid at the low intensity and more rapid at higher intensities.

Figure 3 also shows that the rats hardly pressed during zero intensity. Because of our interest in assessing whether morphine injections of this dose and time after dosing might generally increase motility,

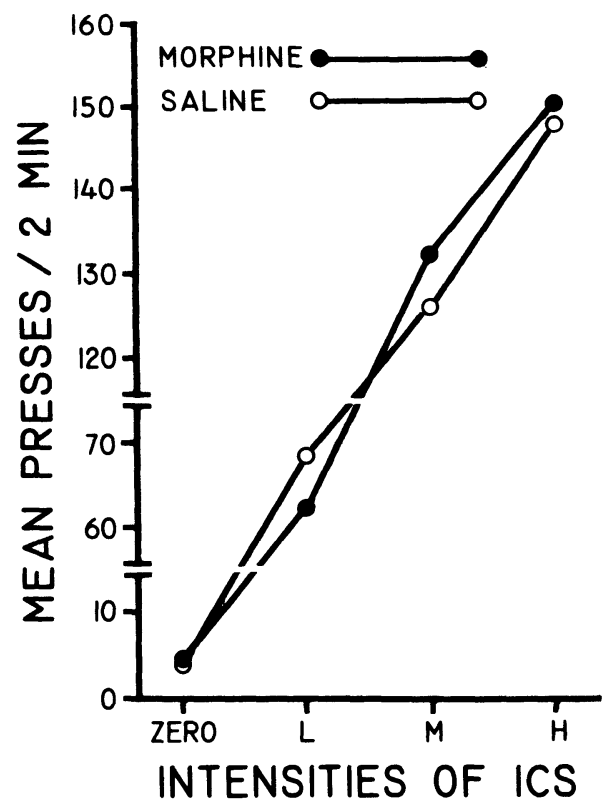

Figure 3. Mean pressing per 2 min during baseline at zero, low, medium, and high intensities of ICS.

a $\mathrm{t}$ test for correlated measures was computed to compare the performance of pressing during baseline and days of injections at zero ICS. The scores submitted to the $t$ for each rat were mean presses of baseline and the mean of the 3 days of injections. Rats of morphine did not reliably increase pressing for zero ICS with morphine injections, $\mathrm{t}(18)=0.8$ (see mean values in Figure 3). Therefore, it is concluded that this dose of morphine does not facilitate general activity that would influence low rates of barpressing. Because the number of presses at zero intensity of ICS was always low and did not shift with morphine injections, further analyses were performed with values only from the low-, medium-, and highintensity conditions.

Figure 4 summarizes the difference scores (score minus mean baseline) of the two groups under the influence of injections. An analysis of variance for a 2 by 3 by 3 factorial design having repeated measures yielded an $F(1,26)$ for the main effect of type of injections $=11.2, \mathrm{p}<.01$, and an $F(2,52)$ for the Injections by Days interaction $=3.54, p<.05$. Other factors were not reliable sources of variance (ps $>.25)$.

Student $t$ tests for correlated measures were performed comparing mean scores of baseline to the comparable score of each day of injections. All ts were sufficiently large to conclude that morphine reliably ( $\mathrm{ps}=.05$ or less) increased pressing at all intensities on all days. The same procedures for the scores of subjects of placebo yielded ts indicating no 


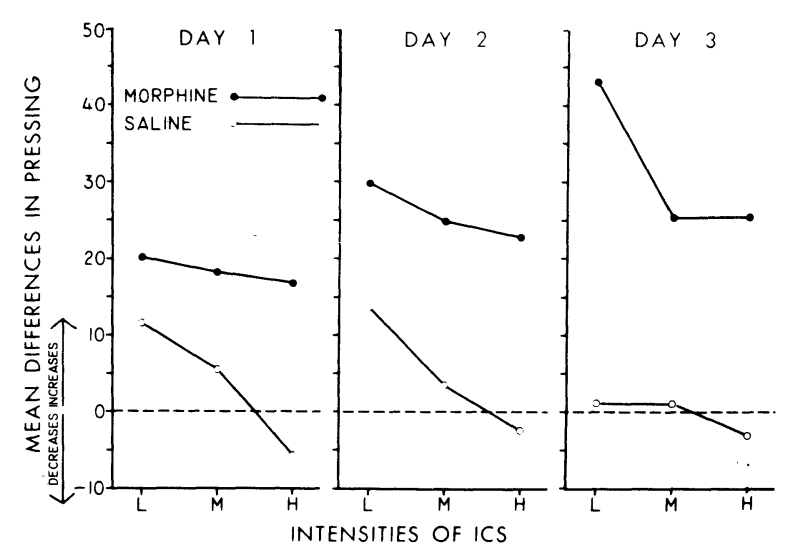

Figure 4. Mean difference scores from baseline of the 3 days of injections.

significant difference between scores of baseline and injections ( $\mathrm{ps}>.30)$.

Direct examination of the brains revealed that all electrode tips were scattered within the lateral hypothalamic area, probably stimulating primarily the MFB and, in some rats, Forel's fields and the zona incerta. Even though there was some difference in placement among the electrode tips, no relationship between the amount of acceleration in pressing under morphine and site of ICS was discernible.

Some subjects of this demonstration were extraordinarily "good pressers," e.g., S-304 pressed 84 times $/ 2$ min during baseline for $5 \mu \mathrm{A}$, its low intensity, and 133 times $/ 2 \min$ for $15 \mu \mathrm{A}$, its high intensity. The rats whose selected intensities were lower than others showed about the same level of acceleration of pressing as did the rats of higher intensities. This observation confirms that morphine does accelerate pressing all along an intensity dimension. Marcus and Kornetsky (1974) reported that morphine reduced lower threshold for intracranial reinforcement. Consequently, there is now ample evidence to support the conclusion that morphine can, at certain doses and regimens of dosing, accelerate pressing for ICS across a wide range of intensities of ICS.

\section{GENERAL DISCUSSION}

Morphine can accelerate pressing for ICS, as described in the introduction, across a wide range of intensities of ICS. Morphine can accelerate pressing for ICS among rats that have sustained some disruption of function due to programming high intensities of ICS. The disruption in function is inferred from the significant reduction in pressing for lowintensity ICS after programming high-intensity ICS.

Magnuson, Tadeusik, and Reid (1976) tested the possibility that morphine might differentially affect responding for ICS, depending on whether the rats were exposed or not to high-intensity ICS. Their data did not support a hypothesis of differential reactivity depending on intensity of ICS. There remains, however, a possibility that in other tests the programming of high-intensity ICS might affect the results.

The results of Experiments 1 and 2 and the results of individual rats of Experiment 2 indicate that there are marked individual differences in reactivity to morphine. Some rats showed marked facilitation of pressing with morphine, while other rats did not. These differences are not apparently related to intensity of ICS. These individual differences evidently demand a larger number per group than used in Experiment 1 . Since the acceleration of pressing for ICS probably reflects an important action of morphine (Rossi \& Reid, 1976), it is important to study these differences.

\section{REFERENCES}

Adams, W. J., Lorens, S. A., \& Mitchell, C. L. Morphine enhances lateral hypothalamic self-stimulation in the rat. Proceedings of the Society of Experimental Biology and Medicine, 1972, 140, 770-771.

Bush, H. D., Bush, M. F., Miller, M. A., \& Reid, L. D. Addictive agents and intracranial stimulation: Daily morphine and lateral hypothalamic self-stimulation. Physiological Psychology, 1976, 4, 79-85.

Collier, H. O. J. Humoral transmitters, supersensitivity, receptors, and dependence. In H. Steinburg (Ed.), Scientific basis of drug dependence. London: Churchill, 1969.

Ellman, S. J., Ackerman, R. F., Bodnar, R. J., Jackler, F., \& Steiner, S. S. Comparison of behaviors elicited by electrical brain stimulation in dorsal brain stem and hypothalamus of rats. Journal of Comparative and Physiological Psychology, 1975, 88, 816-828.

Lorens, S. A., \& Mitchell, C. L. Influence of morphine on lateral hypothalamus self-stimulation in the rat. Psychopharmacologia, 1973, 32. 271-277.

Magnuson, D. J., Tadeusik, C. J., \& Reid, L. D. Addictive agents and intracranial stimulation: Self-stimulation under morphine, amphetamine, and chlorpromazine. Bulletin of the Psychonomic Society, 1976, 8, 459-464.

Marcus, R., \& Kornetsky, C. Negative and positive intracranial reinforcement thresholds: Effects of morphine. Psychopharmacologia, 1974, 38, 1-13.

OLDS, J. The central nervous system and reinforcement of behavior. American Psychologist, 1969, 24, 114-132.

Olds, J., \& Travis, R. P. Effects of chlorpromazine, meprobamate, pentobarbital, and morphine on self-stimulation. Journal of Pharmacology and Experimental Therapeutics, 1960, 128, 397-404.

Pert, A., \& Hulsebus, R. C. Opiate induced facilitation of self-stimulation behavior in the rat. Program and Abstracts of the Society for Neuroscience, 1974. Pp. 372. (Abstract)

Rossi, N., \& ReID, L. D. Affective states associated with morphine injections. Physiological Psychology, 1976, 4, 269-274.

TERANDO, L. Effects of morphine thresholds for intracranial reinforcement. Unpublished Master's thesis, Bradley University, 1974.

VAlenstein, E. S., \& Beer, B. Unipolar and bipolar electrodes in self-stimulation experiments. American Journal of Physiology, 1961, 201, 1181-1186.

(Received for publication February 1, 1977; revision accepted November 18, 1977.) 